\title{
Manufacturing of molds by multiphoton polymerization for micro- replication of optically enhanced surfaces
}

Nerea Otero, Sara Vidal, Félix Ares, Ivette Coto, Francisco Gontad, et al.

Nerea Otero, Sara Vidal, Félix Ares, Ivette Coto, Francisco Gontad, Tamara Delgado, Pablo Romero, "Manufacturing of molds by multiphoton polymerization for micro-replication of optically enhanced surfaces," Proc. SPIE 11271, Laser 3D Manufacturing VII, 112710X (2 March 2020); doi: $10.1117 / 12.2549961$

SPIE. Event: SPIE LASE, 2020, San Francisco, California, United States 


\title{
Manufacturing of molds by multiphoton polymerization for micro- replication of optically enhanced surfaces
}

\author{
Nerea Otero*a, Sara Vidal ${ }^{\text {a }}$, Félix Ares ${ }^{\text {a, }}$ Ivette Coto ${ }^{\text {a }}$, Francisco Gontad ${ }^{\text {a }}$, Tamara Delgado ${ }^{\text {a }}$, Pablo \\ Romero $^{\text {a }}$ \\ a AIMEN - Laser Applications Centre, Polígono Industrial de Cataboi SUR-PPI-2 (Sector 2) Parcela \\ 3. 36418 O Porriño (Pontevedra, Spain)
}

\begin{abstract}
Freeform and microstructured features are generated by MultiPhoton Polymerization with an ultrafast laser, on tool-steel inserts, for microreplication of optical surfaces. The generation of optical surfaces has been studied by combining laser machining and multiphoton polymerization techniques. Resolution in the range of few microns, down to $300 \mathrm{~nm}$ lateral dimension, has been targeted on the optically enhanced surfaces. The microstructured surfaces have been further replicated by injection molding on polymer components of several $\mathrm{cm}^{2}$. The fabrication accuracy and precision has been evaluated in terms of lateral and vertical resolution of the laser generated features, and the replicated ones. The technology aims to simplify the assembly routes for heterogeneously integrated optoelectronics through direct overmolding of the optics on the components (transceivers, LEDs or sensors), with drastic improvements in cost, productivity and performance.
\end{abstract}

Keywords: Multiphoton polymerization, femtosecond laser, replication, injection molding, optical surfaces.

\section{INTRODUCTION}

Optical nano-structures are gaining enormous interest lately, due to their potential to overcome the limits of conventional optics, namely dispersion, chromatic aberration, diffraction limit or diffraction angle ${ }^{1}$. Diffractive optics are being used as the solution for compact, thin and lightweight alternatives to standard optical systems, but the interest is shifting from incremental improvements of existing optics, towards high efficiency nanostructures that can handle phase, amplitude and polarization of the input light in extremely precise ways. While designs of the arrangements of nanoscatterers have been proposed, manufacturing them in a cost-effective way is still challenging, as they require to write arbitrary structures, often with high aspect ratio and sharp edges (phase shifts) with few wavelengths of lateral resolution and sub-wavelength vertical accuracy.

Multi-Photon Polymerization (MPP) has been demonstrated as reliable technique for the fabrication of complicated 3D structures in photosensitive materials ${ }^{2}$. This technique has been successfully used for the manufacturing of different structures for different applications $\mathrm{s}^{3-4}$ as for example the fabrication of 3D scaffolds for applications in tissue engineering ${ }^{5}$. The main concept behind the MPP technique is the use of focused laser radiation to trigger a polymerization reaction inside a volume of resin. The fabricated part is self-supported in this fabrication media (photosensitive resin), so a laser beam can write a structure in 3D with no need of support structures or layer-by-layer approach.

This paper explores the use of MPP for fabricating microstructured surfaces on inserts that will be further used through injection molding (IM) process for replication of freeform optical surfaces, targeting $>1 \mu \mathrm{m}$ lateral accuracy and vertical replication fidelity (reproduction of the nanoroughness) under $100 \mathrm{~nm}$. The experimental work has been developed within the H2020 project FLOIM, which explores the generation of complex optical functions through replication of nanostructured mould surfaces, which can produce controlled diffractive effects -from lensing to wavelength splittingextending the design space for extraordinary innovation potential and miniaturization. The main objective is to develop new, flexible and innovative process chains to handle complex designs that include opto-electronic functionalities, through the development of an integrated functional embedding technology based on thermoplastic IM.

*notero@aimen.es; phone +34 986344 000; www.aimen.es; www.floimproject.eu

Laser 3D Manufacturing VII, edited by Bo Gu, Hongqiang Chen, Henry Helvajian, Proc. of SPIE

Vol. 11271, 112710X · (c) The Authors. Published under a Creative Commons

Attribution CC-BY 3.0 License · doi: 10.1117/12.2549961

Proc. of SPIE Vol. 11271 112710X-1 


\section{EXPERIMENTAL SETUP AND PROCEDURE}

\subsection{Samples preparation}

The resin used in these experiments is Ormostamp ${ }^{\circledR}$ (hybrid photoreactive polymer, Micro Resist Technology GmbH), combined with the photoinitiator (PI) Cabazyl (1,3,5-Tris(2-(9-ethylcabazyl-3)ethylene)benzene, SIGMA-ALDRICH). 2 $\mathrm{mg}$ of PI are mixed with $1 \mathrm{ml}$ of DCM (Dichloromethane) by means of a magnetic stirrer for 5 minutes, $1 \mathrm{~g}$ of Ormostamp ${ }^{\circledR}$ is then added, and the mixture is stirred for 2 hours. In order to evaporate the rest of the DCM, the mixture is placed in a fume hood for at least 8 hours. The process is performed under red light illumination to avoid undesired polymerization.

The material selected as insert substrate is P20 mold steel, a common material used for plastic injection mold cavities. The inserts, whose dimensions are $150 \times 40 \times 2 \mathrm{~mm}$, are polished previously to their use as substrates for MPP. Two different methods have been tried in order to improve the adhesion of the polymerized microstructures to the inserts, with and without an additional flat Ormostamp ${ }^{\circledR}$ extra layer:

Method 1: (1) polished steel inserts are cleaned with acetone and isopropanol before being used; (2) a thin layer of the prepared resin is deposited on one side of the steel insert, by using a Zehntner ZAA2300 doctor blade automatic coater at $3 \mathrm{~mm} / \mathrm{s}$, with $50 \mu \mathrm{l}$ of the prepared resin, providing a $30 \mu \mathrm{m}$ thickness film before curing.

Method 2: (1) polished steel inserts are cleaned with acetone and isopropanol before being used; (2) sample is heated at $200^{\circ} \mathrm{C}$ for 5 minutes; (3) the sample is cooled to room temperature before depositing a thin layer $(30 \mu \mathrm{m})$ of pure Ormostamp on one side of the steel insert as in method 1; (4) sample is heated at $80^{\circ} \mathrm{C}$ for 2 minutes; (5) the thin layer of the deposited resin is cured with an UV lamp for 1 minute; (6) the sample is heated at $130{ }^{\circ} \mathrm{C}$ for 10 minutes; (7) a thin layer of the prepared resin is deposited on top of the cured layer, following the same procedure as step (2) in method 1.

\subsection{Experimental Setup}

The system used for the MPP process is an OPTEC micromachining station which integrates a laser source Satsuma HP ${ }^{2}$ (Amplitude Systèmes), emitting at a visible wavelength $(515 \mathrm{~nm})$, with micrometric precision XYZ linear stages (Aerotech). The laser beam is focused with an PLAPON60XO Olympus microscope objective with nominal 60X magnification. To obtain adequate beam distribution, a $4 \mathrm{x}$ beam expander was used to reach a beam diameter of $4 \mathrm{~mm}$ of the collimated beam at the microscope objective entrance. The setup is represented in Figure 1 (left). The process is performed under red light illumination to avoid undesired polymerization of the resin.
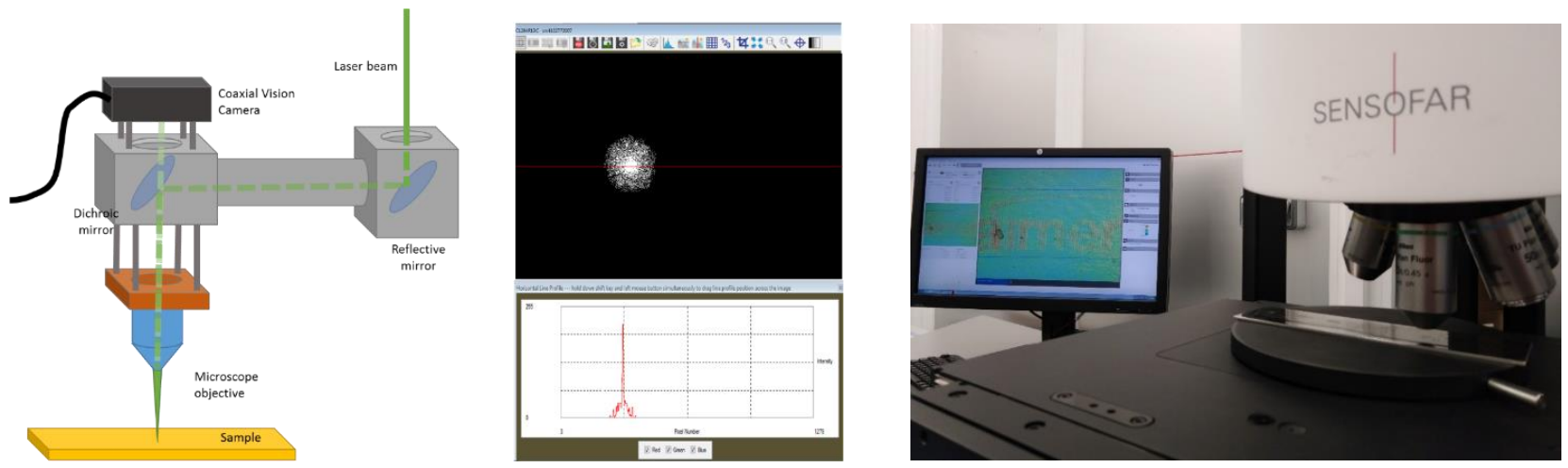

Figure 1. (left): Photopolymerizing system with integrated coaxial camera. (right): confocal profilometer system at AIMEN.

Different geometries were designed in CAD and then fabricated by MPP of the as deposited resin coating the steel insert. The laser beam was focused inside the resin with an energy under $10 \mathrm{~nJ}$ and at a $500 \mathrm{kHz}$ repetition rate, following the path corresponding to the previously prepared CAD geometries. Immediately after the fabrication, the inserts were submerged in a bath of Methyl Isobutyl Ketone (MIBK) for 30 minutes, and then rinsed in an Isopropanol bath for 10 minutes, in order to eliminate the not polymerized resin. The structures were measured before and after the replication process by using a confocal profilometer S-Neox (Sensofar), Figure 1 right.

\subsection{Replication process}

The injection molding process was performed with an ENGEL injection molding machine, model VICTORY 40. The material chosen for the replication of the microstructures on the insert surface is Zylar ${ }^{\circledR}$ 960, which is a high flow 
transparent styrene acrylic copolymer that provides toughness equivalent to some grades of polycarbonate, but can be processed at lower temperatures (around $200^{\circ} \mathrm{C}$ ). Using lower processing temperature extends the life of the inserts, and additionally, avoids accidental damages on the electric components of the optoelectronic devices during the IM. The IM was performed by following the technical recommendations of the material manufacturer.

\section{RESULTS AND DISCUSSION}

Adhesion and replication of the polymerized samples following the two different methods explained in section 2.1 were evaluated.

\subsection{Method 1}

Results obtained following Method 1 showed poor adhesion of the photopolymerized resin to the polished steel insert. In this method, lines were fabricated at different angles in a star-like geometry. Figure 2 shows the difference between a structure partially attached to the surface and another structure that got completely detached from the insert and collapsing over itself. No replication was done in this case, due to the lack of good adhesion of the polymerized structures.



Figure 2. Photopolymerized resin forming a star-like geometry.

\subsection{Method 2}

In Method 2, a layer of pure Ormostamp ${ }^{\circledR}$ is applied to the steel insert and cured before applying the resin+photoinitiator mix. This first layer improves drastically the adhesion of the photopolymerized microstructures to the steel insert, as the attachment is not produced directly to the steel insert, but to the pure Ormostamp ${ }^{\circledR}$ layer.

Several groups of parallel lines were photopolymerized using this method (Figure 3), as well as several AIMEN logos. Different groups of lines were used to evaluate the adhesion of the photopolymerized structures and optimize the process parameters.

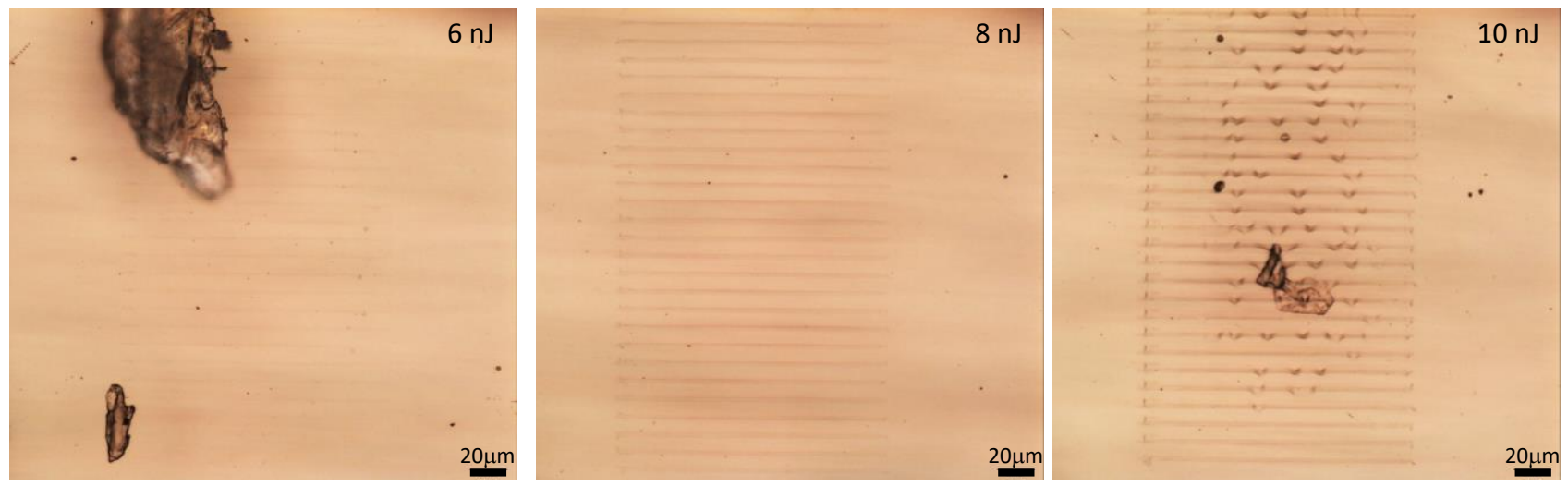

Figure 3. Groups of photopolymerized lines varying laser pulse energy, from $6 \mathrm{~nJ}$ per pulse to $10 \mathrm{~nJ}$

As it can be seen in Figure 3, the suitable laser pulse energy for photopolymerization was found to be in the range between 6 and $10 \mathrm{~nJ}$. The photopolymerization threshold was found at $6 \mathrm{~nJ}$, under the above-mentioned experimental conditions. while pulse energies over $10 \mathrm{~nJ}$ resulted in the appearance of undesired bubbles inside the resin, a well-known consequence of excessive radiation doses of Ormocer ${ }^{\circledR}$ resins. 
Several AIMEN logos were photopolymerized and replicated by injection molding, with the processing parameters optimized for line writing (8nJ/pulse @ 500kHz), as shown in Figure 4. Writing energy and overlap is high enough to produce lines of 5 microns in width, as can be seen in such figure. The distance between the hatch lines is $2 \mu \mathrm{m}$, so there is overlapping between them, and the structure is created as a solid block, as shown in Figure 5, with a maximum roughness around $200 \mathrm{~nm}$. In this way, a compromise between fabrication speed and film roughness was made in order to be able to fabricate the master on large areas with a roughness that could be adequate for the target application.

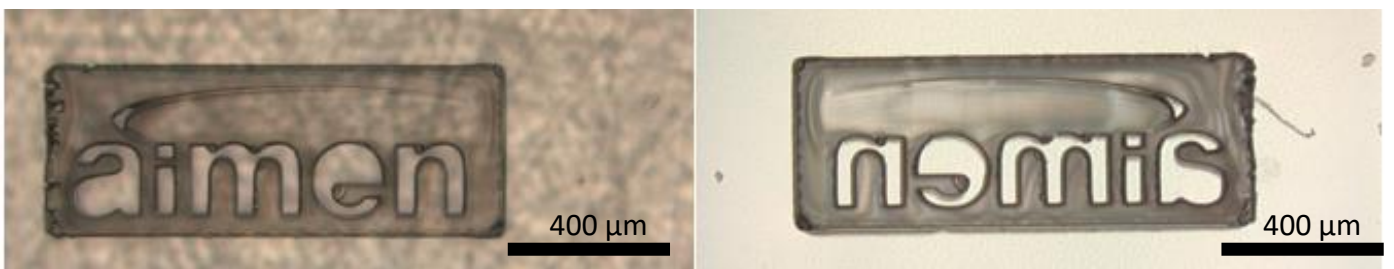

Figure 4. Photopolymerized AIMEN logo contour (left) and injection molded replica of the polymerized logo (right).

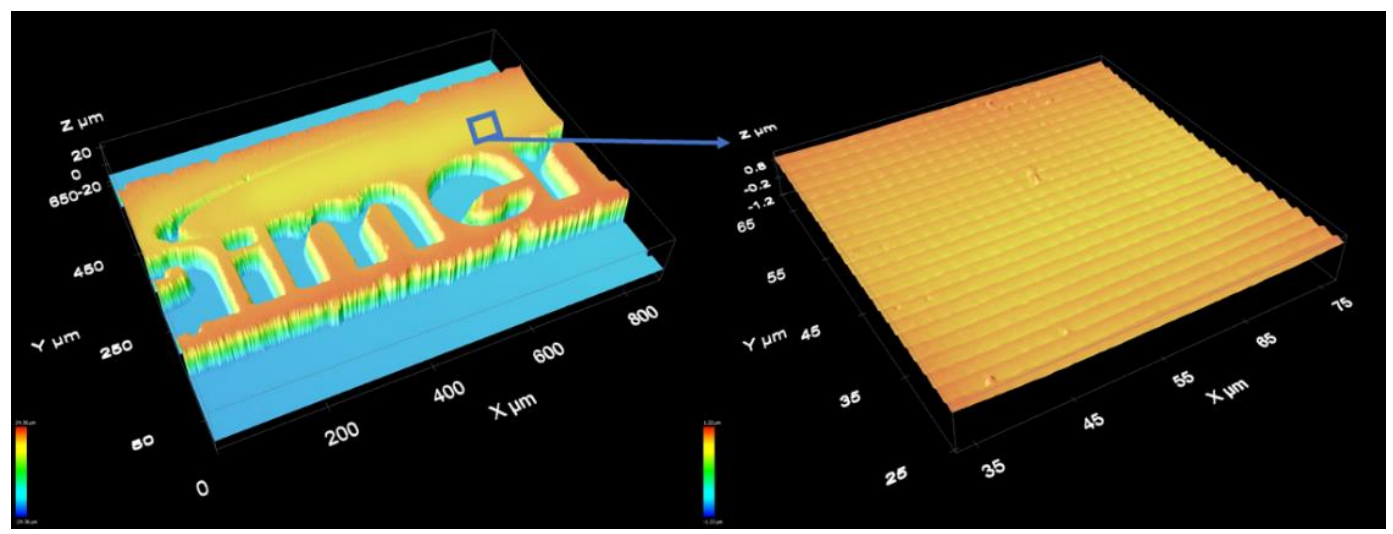

Figure 5. 3D topography of the photopolymerized AIMEN logo on a steel insert (left), where overlapping of the parallel lines in the hatching can be appreciated (right).

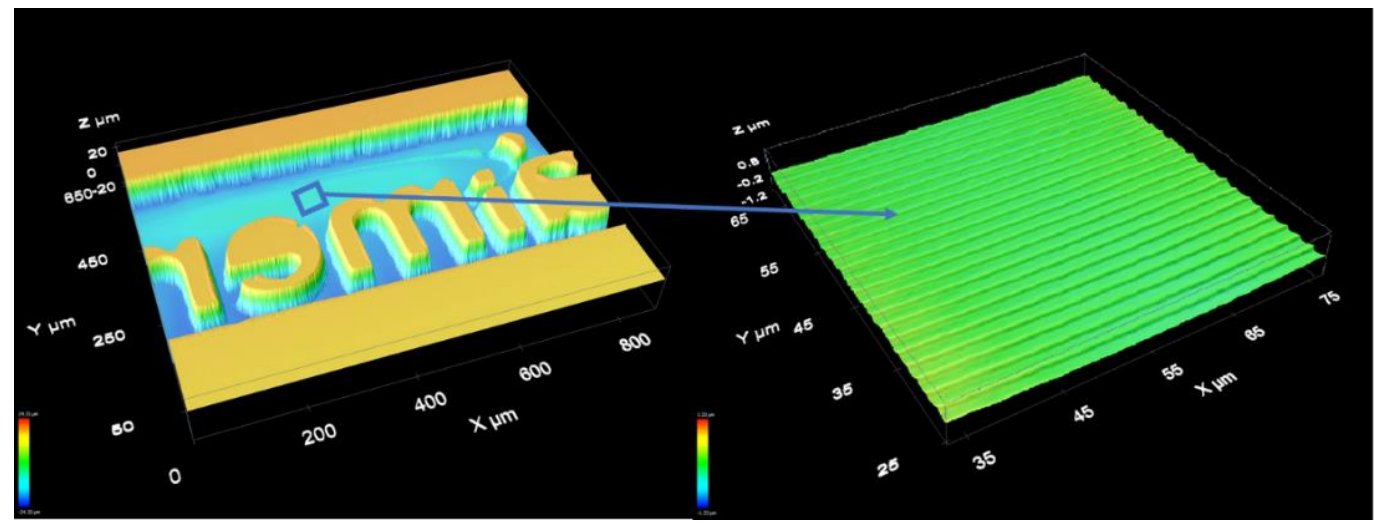

Figure 6. 3D topography of the injection molded replica of an AIMEN logo. The lines overlapping can still be appreciated.

Replication of polymerized structures was made by the injection molding process described in section 2.3, using Zylar® 960 as injection material. The results obtained from these trials show a good quality of replication (Figure 6), with details as small as $0.5 \mu \mathrm{m}$ perfectly reproduced in lateral resolution. As expected, the maximum roughness of the replicated sample was smoother than the equivalent in the original insert $(130 \mathrm{~nm}$ instead of $200 \mathrm{~nm})$, indicating that a correction of the insert roughness would not be necessary. However, the pitch between the hatch lines should be optimized when the application needed for lower roughness, with a cost in terms of fabrication speed. 
More than 50 replicas were made by injection molding and no damage to the insert is detected, which means that photopolymerized Ormostamp ${ }^{\circledR}$ on tool steel inserts is a good choice for applications that require Injection Molding replication of microstructures with high resolution.

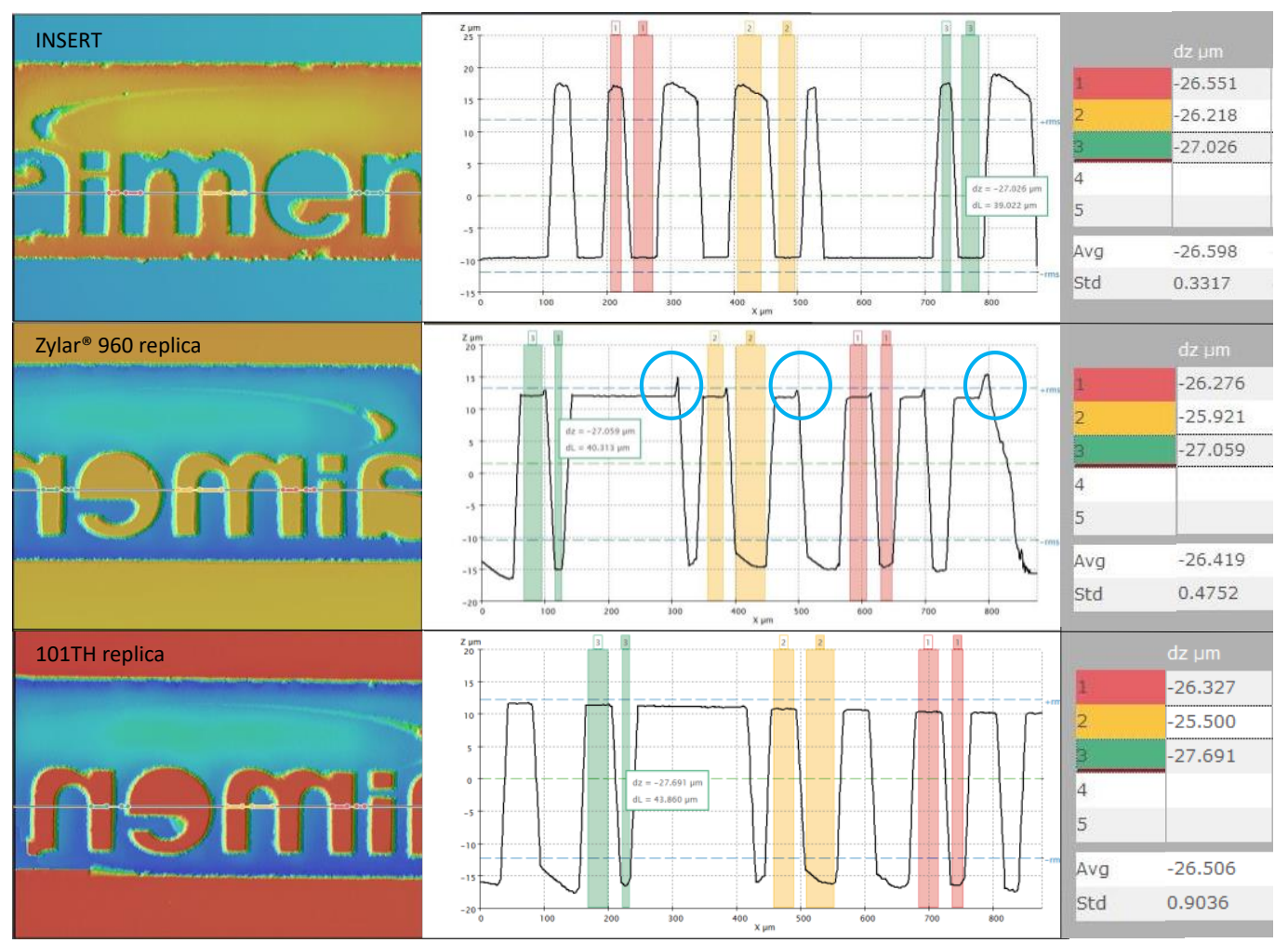

Figure 7. Topographic profile of (a) a photopolymerized insert, (b) an injection molded replica, (c) a 101TH replica.

However, even if the injected replicas show very good quality, some defects appear on the edge of the structures (highlighted in light blue, Figure 7), when measured with the optical profilometer, in the form of sharp peaks of about 1 micron in height, suggesting problems with the filling and demolding process during the injection cycle of the Zylar® 960 material. To confirm this hypothesis, a high resolution replication material, 101TH (Thixotropic silicone, Microset), was used to faithfully replicate the polymerized structures (Figure 8). This replica was measured and compared with the injected ones, in order to confirm that the polymerized features were not damaged during the injection process (Figure 7).
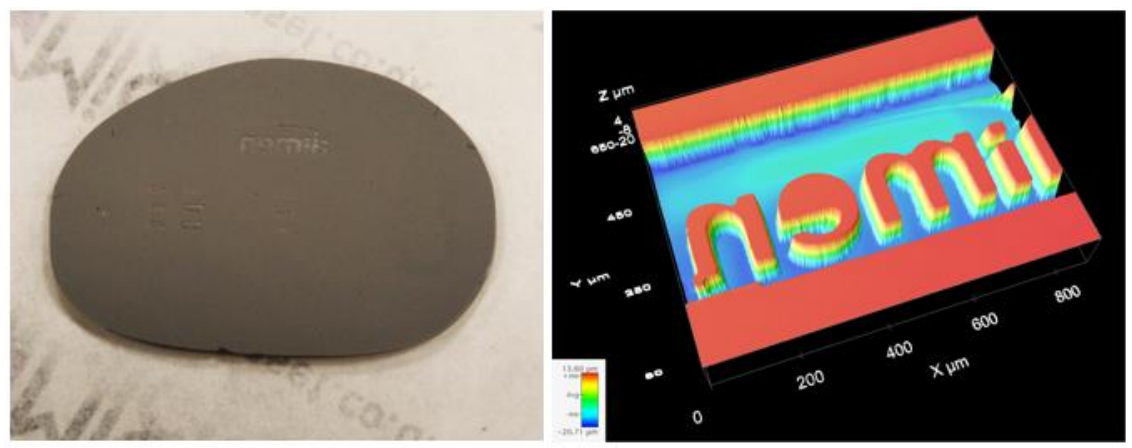

Figure 8. AIMEN logo replicated in high resolution replicating silicone 101TH.

Figure 7 shows that the 101TH replica does not possess the same defects as the injection molded replica in the edges, indicating that the origin of the defect is either in injection molding or caused by the demolding. Outside these edge defects, the high resolution replication silicone obtains similar geometries than the injection molding copolymer, which suggests that the injection molding process induces this defect. 


\section{CONCLUSIONS}

Obtained results validated multi photon polymerization as a viable technique for microstructuring of injection molding inserts by using Ormostamp ${ }^{\circledR}$ as coating resin with good adhesion to the steel inserts. Micro and nano-structures were successfully replicated on Zylar ${ }^{\circledR} 960$ samples, demonstrating that optically functional surfaces can be fabricated by the described procedures. The microstructured moulds have shown good behavior during the IM, surviving several tens of IM cycles at industrial conditions.

Nevertheless, due to the flowing grade of Zylar ${ }^{\circledR} 960$, sharp features fabricated on the insert surface are not properly replicated during the IM process. Further effort should be paid for selecting alternative polymers with best flowing properties (higher melt flow rate -MFR-, or higher melt flow index - MFI-) and/or different injection molding process parameters which should guarantee better replication results.

\section{ACKNOWLEDGEMENTS}

This work has received funding from the Europeans Union's Horizon 2020 research and innovation programme under grant agreement $n^{\circ} 820661$.

The authors would like to thank the FLOIM consortium for their collaboration in the project: UPC, PROMOLDING, HYBRONICS, MONDRAGON ASSEMBLY, FAGOR AUTOMATION, ADAMA INNOVATIONS, FLEXENABLE, RECENDT, FRAUNHOFER IWU, CEIT-IK4 and SNELL OPTIQUES.

\section{REFERENCES}

[1] Wang, C. Wang, et al., "Mat. and Lenses for Optical Lithography beyond the Diffraction Limit", Plasmonic Struct., (2016). doi:10.3390/mi7070118

[2] Gontad, J., Vidal, S., Otero, N., Romero, P., "Massive Parallelization of Laser Beams with Diffractive Optical Elements for High Speed Two Photon Polymerization" Lasers in Manufacturing Conference, (2019)

[3] Serbin, J. et al., "Femtosecond laser-induced two-photon polymerization of inorganic-organic hybrid materials for applications in photonics", Optics Letters 28, p. 301, (2003)

[4] Waheed, S. et al., "3D printed microfluidic devices: enablers and barriers", Lab on a Chip 16, p. 1993, (2016)

[5] Ovsianikov, A., et al., "Laser Fabrication of three-dimensional CAD scaffolds from photosensitive gelatin applications in tissue engineering" Biomacromolecules 12, p.851. (2011) 